\title{
Flow Characteristics and Structure Optimization of a Baffle Plate Heat Exchanger
}

\author{
Mengshi Lu ${ }^{1}$, Zhuoheng Feng ${ }^{1}$, Yungang Wang ${ }^{1}$, Hongwei Yang ${ }^{1}$, Wenqing Xia ${ }^{1}$, \\ Haimiao Zhu ${ }^{1}$ and Dan Hua ${ }^{2 *}$
}

${ }^{1}$ Everbright Environmental Technology (China) Co.,LTD, Nanjing, China, ${ }^{2}$ Key Laboratory of Energy Thermal Conversion and Control of Ministry of Education, School of Environmental Science and Engineering, Suzhou University of Science and Technology, Nanjing, China

In this study, a theoretical model of the hot and cold fluid flow, heat transfer, and thermal stress coupling in a baffle plate heat exchanger is established, and numerical simulation is carried out. The flow field in the heat exchanger and the deformation of the heat exchange tube bundles in the baffle plate heat exchanger are studied. The optimization effect of the diversion grid on the flow field at the inlet of the variable section is emphasized. The influence of grid arrangement on the flow field and the performance of the tube bundles are

OPEN ACCESS

Edited by:

Chengbin Zhang,

Southeast University, China

Reviewed by:

Chaoqun Shen,

Yangzhou University, China

Dongyan Gao,

Naniing Institute of Technology (NJIT),

China

*Correspondence:

Dan Hua

huadan@usts.edu.cn

Specialty section:

This article was submitted to

Process and Energy Systems

Engineering,

a section of the journal

Frontiers in Energy Research

Received: 06 December 2021

Accepted: 20 December 2021

Published: 31 January 2022

Citation:

Lu M, Feng Z, Wang $Y$, Yang $H$, Xia $W$,

Zhu H and Hua D (2022) Flow Characteristics and Structure

Optimization of a Baffle Plate

Heat Exchanger.

Front. Energy Res. 9:830056.

doi: 10.3389/fenrg.2021.830056 analyzed and discussed. The results show that the backflow situation can be effectively improved and the vortex intensity in the straight section of the cold fluid inlet can be greatly reduced by adding the grid. The deformation of the heat exchange tube bundles illustrates the maximum deformation with $1.39 \mathrm{~mm}$ at the position of the strongest backflow. The deformation can be reduced by $26 \%$ due to the arrangement of the diversion grid, which is helpful to improve the safety of the device.

Keywords: baffle plate, heat exchanger, GRID, vortex flow, deformation

\section{INTRODUCTION}

Generally speaking, heat transfer enhancement can be divided into passive enhancement and active enhancement. The former is more preferred in practical engineering since active heat transfer enhancement needs to introduce a control system and consume energy. The passive strengthening of a heat exchanger mainly includes in the tube and baffle strengthening. A special-shaped tube is mainly used in tube enhancement instead of a straight tube to stimulate the degree of fluid turbulence to enhance heat transfer. Baffle enhancement is very important to improve the shell side heat transfer of the heat exchanger, which is widely used because it can achieve a high heat transfer film coefficient at the shell side, with advantages of simple structure, easy production, and installation, but it has retention area and have disadvantages of easy scaling on the shell side and a great pressure drop along the way. In this context, it is urgent to carry out the structural design optimization of the baffle plate heat exchanger.

In the baffle plate heat exchanger, due to the variable cross-section or bend at the inlet, it is easy to form a vortex, resulting in uneven flow field distribution and reduced efficiency and increased resistance of the heat exchanger (Blecich, 2015). In addition, the exciting force caused by vortex shedding is easy to cause vibrations of the heat exchange tube and fatigue damage of the tube bundle (Wang, 2015). Therefore, measures must be taken to improve the non-uniformity of the flow field. The flow field uniformity inside the heat exchanger is generally improved by installing a diversion device to enhance the heat exchange effect in engineering applications (Long, 2014). Yang et al. (2019) took the tubular GGH of a power plant as the research object and installed deflectors at the 

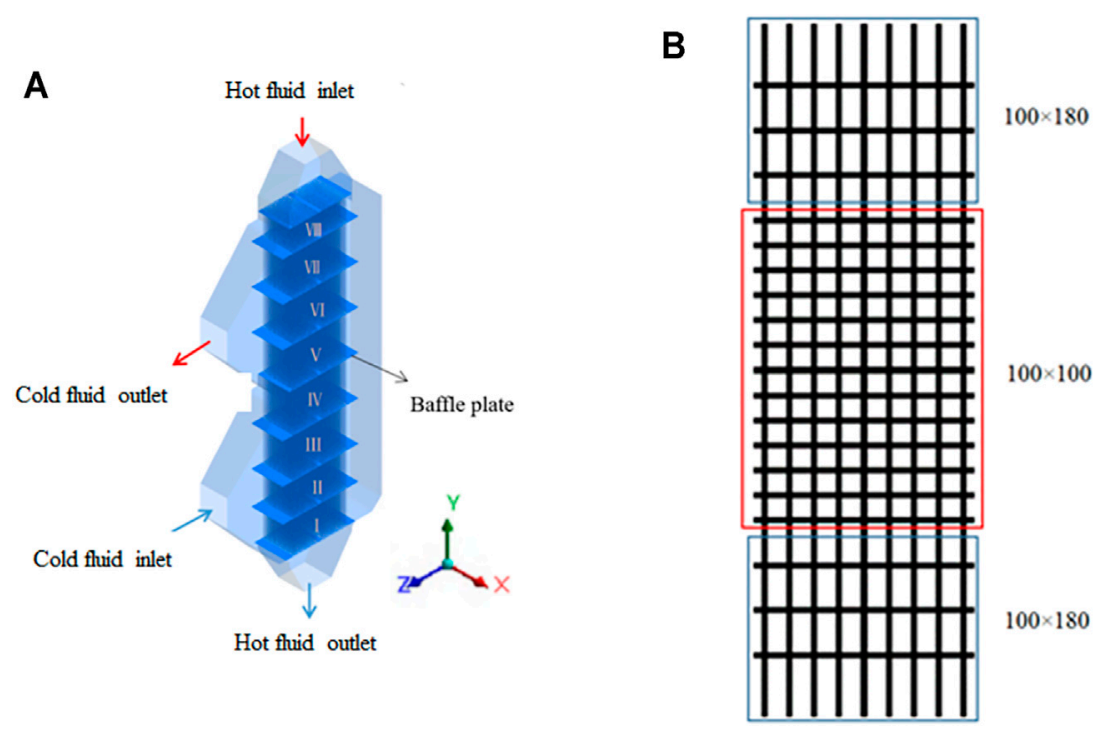

FIGURE 1 | Diagram of model: (A) baffle plate heat exchanger and (B) grid.

inlet of the cold and hot fluid, respectively. It was found that the reasonably designed deflectors can restrain the uneven distribution of the flow field caused by elbow and variable sections. Wu et al. (2020) simulated the inlet flow channel of original flue gas and net flue gas of the $600 \mathrm{MW}$ coal-fired unit and optimized the flow field by installing the diversion device to reduce the unevenness to within \pm 0.2 . Liu et al. (2017) conducted a simulation study on the influence of a deflector and air distributor on the uniformity of the flue channel with a variable section and found that the installation of the deflector and air distributor can reduce the standard deviation of velocity distribution in the channel by $30.3 \%$. At present, the research on the influence of a deflector on a heat exchanger mainly focuses on the influence of non-uniformity of the flow field, and its influence on the heat exchange tube bundle mainly focuses on the vibration of the tube bundle (Chen, 1987; Miao, 2016; Shi et al., 2019). It should be pointed out that the corresponding heat exchange tube bundle in the heat exchanger will deform to a certain extent under the action of an uneven flow field. The fluorine plastic heat exchanger, in particular, compared with a normal heat exchanger, its elastic modulus is smaller and easier to deform under jet impact. However, there are few reports on the influence of diversion devices on the deformation of the heat exchange tube bundle in the existing research.

Taking the fluorine plastic baffle plate heat exchanger as the research object, the flow field at the inlet of the heat exchanger is numerically simulated; in this study, the flow field at the inlet is optimized by installing a grid; the deformation of the heat exchange tube bundle in the baffle plate heat exchanger is calculated after installing a grid, and the flow field and the deformation of the heat exchange tube bundle before and after optimization are compared and analyzed.

\section{CALCULATION SETTINGS}

\section{Geometric Model}

Based on the real structure and size of the baffle plate heat exchanger in engineering, the calculation model of the same size as real structure is established, as shown in Figure 1A. Heat is exchanged between the hot fluid and cold fluid in the heat exchanger. The hot fluid flows along the pipe side, from upper part of the baffle plate heat exchanger, through the heat exchange tube bundles, and then flows from the lower end; the cold fluid, along the shell side, which enters the heat exchanger from the lower trumpet and flows out from the upper trumpet. The baffle plate inside the heat exchanger separates the cold fluid into 8 channels, numbered I-VIII from bottom to top. The baffle plate not only plays a conductivity role on the fluid but also plays a limited role on the heat exchange tube beam. The heat exchanger is composed of 20 rows ( $Z$ direction) and 13 rows ( $X$ direction) of 260 heat exchange tubes, the inner diameter of which is $50 \mathrm{~mm}$ and the thickness is $1 \mathrm{~mm}$. The outer wall of the heat exchange tube is $26 \mathrm{~mm}$ in the $Z$ direction and $20 \mathrm{~mm}$ in the $X$ direction, and the total heat exchange area of the device is about $340 \mathrm{~m}^{2}$.

The grid is arranged at the cold fluid inlet, the distance between which is $700 \mathrm{~mm}$, and the interval between the vertical grid panel is $100 \mathrm{~mm}$; the interval between the internal transverse grid panel is $100 \mathrm{~mm}$, and the interval between the lateral grid panel is $180 \mathrm{~mm}$, that is, the interval between the grid panel is small in the middle and large on both sides, as shown in Figure 1B. It helps improve the resistance in the middle of the guide grille; moreover, the fluid in the jet area will flow to the surrounding areas with low resistance, which effectively diverts the fluid. 


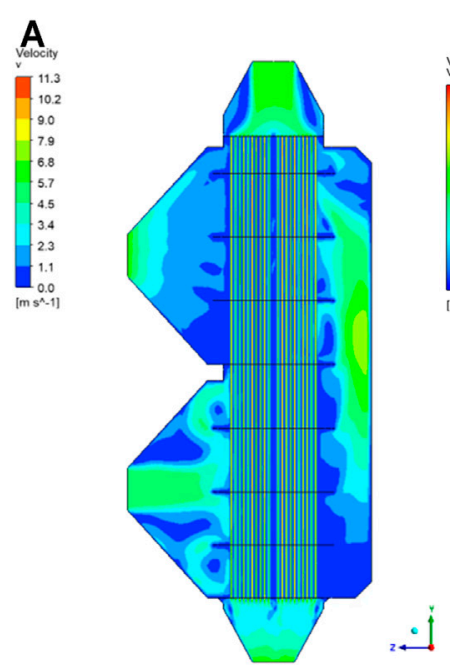

(i)

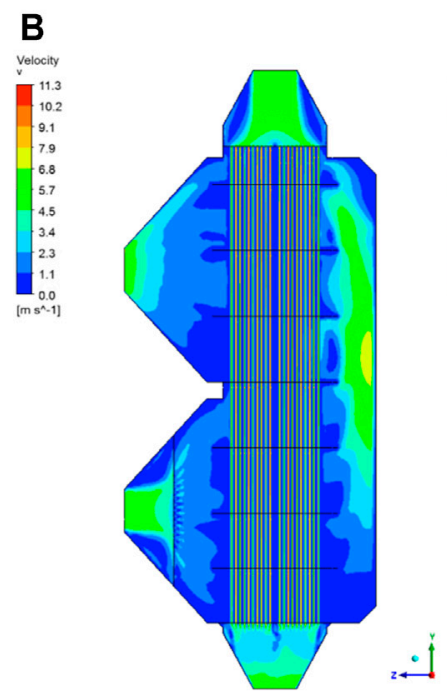

(i)
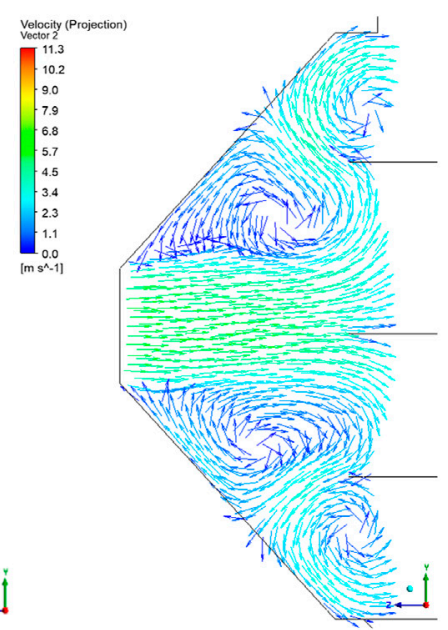

(ii)

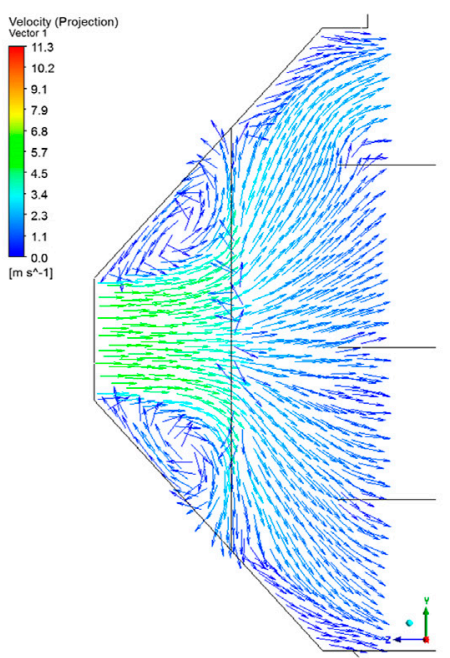

(ii)

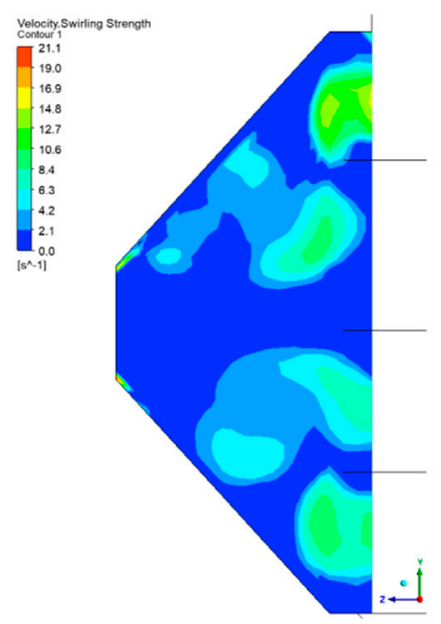

(iii)

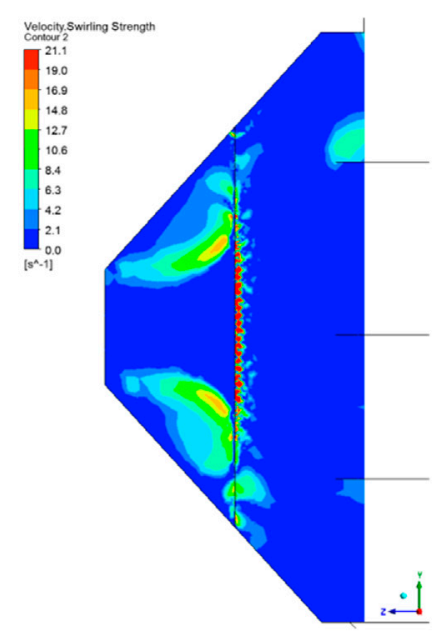

(iii)

FIGURE 2 | Flow field distribution: (A) without grid and (B) with grid. (i) Velocity cloud, (ii) Velocity vector of the cold fluid inlet, (iii) Vortex intensity of the cold fluid inlet.

\section{Calculation Model}

Fluid flow and heat transfer in the heat exchanger is a typical turbulent flow and heat transfer process in finite space. In this calculation, a three-dimensional steady-state turbulent flow and heat transfer model is adopted, in which the $\mathrm{k}-\varepsilon$ model is used. The SIMPLE algorithm is used to deal with the coupling between pressure and velocity, and a second-order upwind discrete scheme is used to ensure the accuracy of the calculation.

The inlet of the computing domain adopts the pressure inlet boundary, the inlet temperature of the hot fluid and the cold fluid is $140^{\circ} \mathrm{C}$ and $70^{\circ} \mathrm{C}$, respectively. The pressure outlet boundary is adopted at the outlet of the computing domain, and the static pressure at the outlet of the hot fluid and cold fluid is $-5,500 \mathrm{~Pa}$ and $-6,000 \mathrm{~Pa}$, respectively. The fluid volume flow rate is
$10,000 \mathrm{Nm}^{3} / \mathrm{h}$, the tube side flow rate is $8.2 \mathrm{~m} / \mathrm{s}$, and the shell side flow rate is $3.1 \mathrm{~m} / \mathrm{s}$. There is no slip boundary on the inner wall of the heat exchanger, both sides of the heat exchange tube bundle and baffle plate. The adiabatic boundary is adopted on the outer wall of the heat exchanger. The hot and cold fluid are,

TABLE 1 | Average vortex intensity in the straight section of the cold fluid inlet.

\begin{tabular}{lcc}
\hline Position & \multicolumn{2}{c}{ Vortex intensity $\left.\mathbf{( s}^{-\mathbf{1}}\right)$} \\
\cline { 2 - 3 } & Without grid & With grid \\
\hline Middle section & 4.244 & 0.803 \\
1/4 section & 4.170 & 0.773 \\
3/4 section & 4.243 & 0.954
\end{tabular}




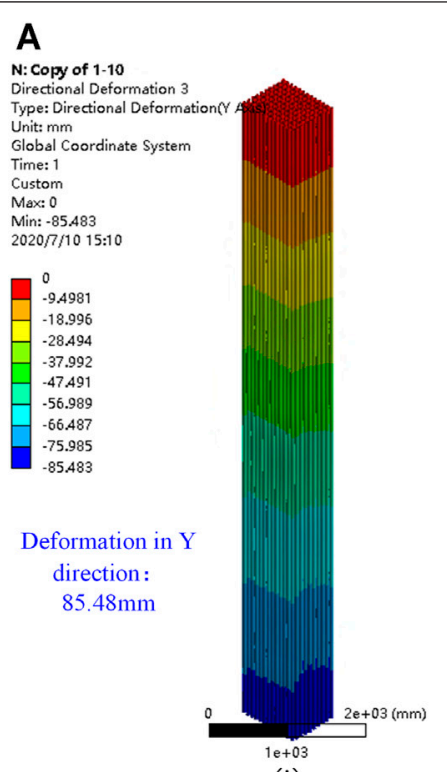

(i)

\section{B}

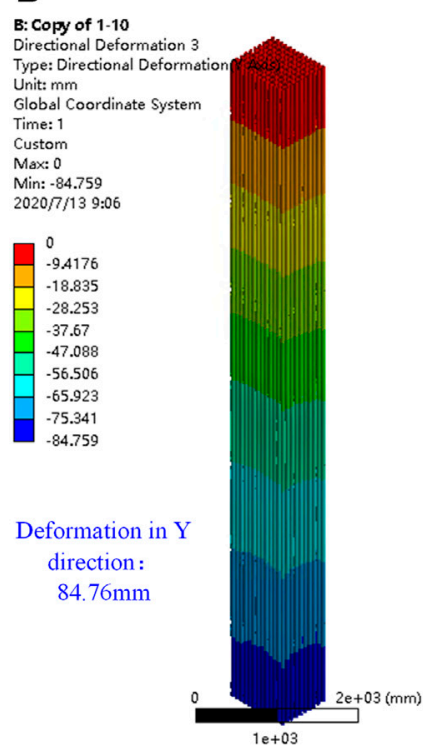

(i)

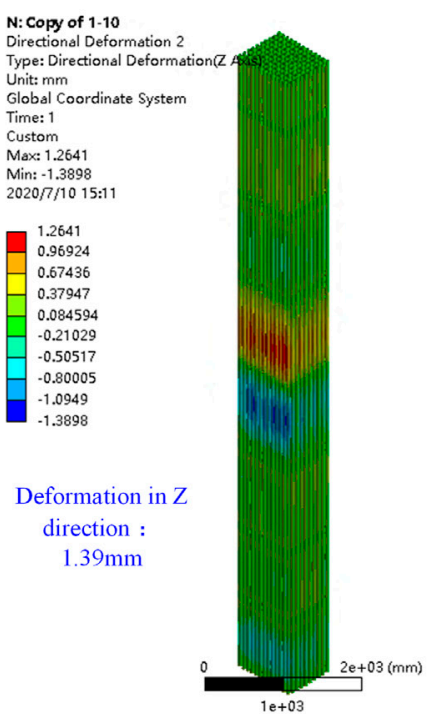

(ii)

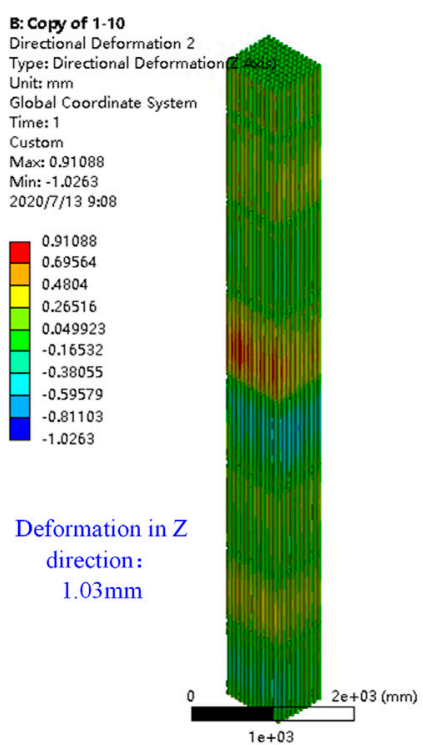

(ii)

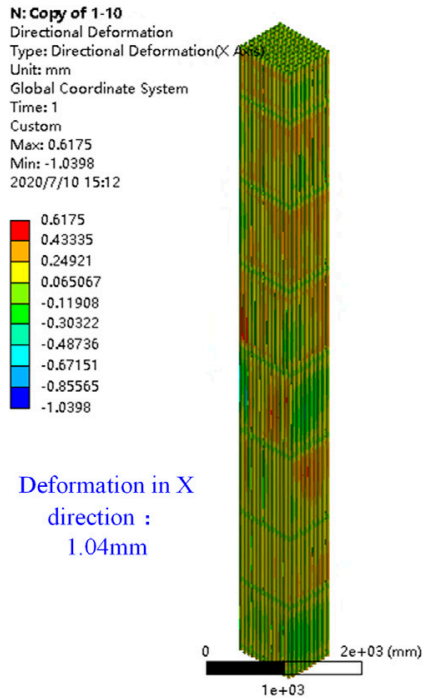

(iii)

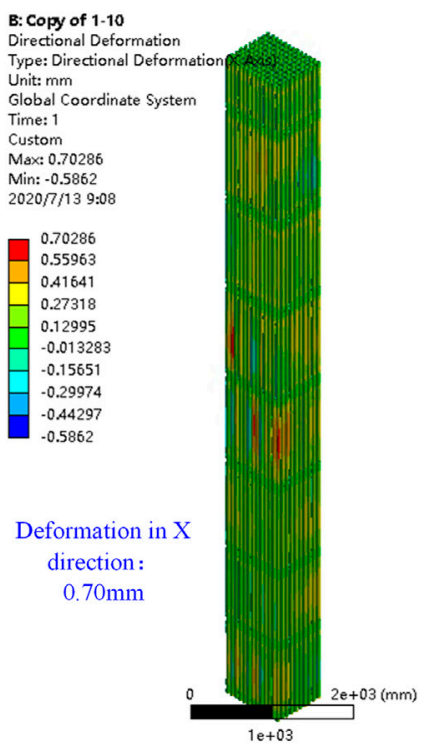

(iii)

FIGURE 3|Deformation of the heat exchange tube bundle: (A) without grid and (B) with grid. (i) Deformation in the $Y$ direction, (ii) Deformation in the $Z$ direction, (iii) Deformation in the $X$ direction.

respectively, on both sides of the heat exchange tube bundle and baffle plate, and the coupling boundary is adopted. The heat transfer coefficient of the tube wall is $0.2 \mathrm{~W} /(\mathrm{m} \mathrm{K})$, the density is $2,190 \mathrm{~kg} / \mathrm{m}^{3}$, the linear expansion coefficient is $1.2 \times 10^{-6} /{ }^{\circ} \mathrm{C}$, the tensile modulus is $280 \mathrm{MPa}$, and the strength is $25 \mathrm{MPa}$. The specific heat capacity, viscosity, thermal conductivity, and other physical parameters of the fluid are related to the temperature of the fluid and are calculated according to the widely used direct calculation method proposed by Xu (1999).

\section{RESULTS AND DISCUSSION}

\section{Influence of the Diversion Grid on the Flow}

\section{Field}

The velocity cloud in the YZ direction, the velocity vector, and the vortex intensity at the inlet of the cold fluid without the grid is shown in Figure 2A. The velocity cloud shows that after the cold fluid enters the heat exchanger from the trumpet-mouth, the cold fluid will form the phenomenon of the jet due to the sudden 
increase of the cross-section area, and the fluid of the jet will absorb the fluid around it, leading to the generation of reflux. The jet flow and reflux at the trumpet-mouth position are shown in Figure 2Aii, and the vortex intensity at the trumpet-mouth is shown in Figure 2Aiii. The stronger the backflow, the greater will be the vortex intensity. As can be seen from the figure, the straight pipe between the trumpet and the heat exchanger has the strongest backflow and the largest vortex intensity, owing to the fact that the position is close to the tube bundle inside the heat exchanger. In order to avoid the fluid backflow on the heat exchange performance and mechanical properties of heat exchange tube bundles, it is necessary to arrange a diversion device in the trumpet section of the cold fluid inlet to optimize the fluid diversion.

Figure 2B shows the flow field distribution when the diversion grid (shown in Figure 1B) is installed $700 \mathrm{~mm}$ away from the inlet of the cold fluid. The cold fluid is quickly shunted after entering the heat exchanger when the grid is arranged, and the flow in each channel at the inlet side tends to be consistent; the reflux situation in the trumpet mouth has been significantly improved, and the backflow at the straight pipe section of the cold fluid inlet is basically eliminated. The vortex intensity is the largest at the grid, and it decreases obviously at the straight pipe between the trumpet and heat exchanger.

In order to better obtain the improvement effect of the grid on the flow field, the average vortex intensity values at the inlet of the straight pipe section of the cold fluid at the middle section in the $\mathrm{YZ}$ direction, $1 / 4$ section in the $\mathrm{YZ}$ direction, and 3/4 section in the $\mathrm{YZ}$ direction were respectively counted, as shown in Table $\mathbf{1 .}$ It can be seen that after the addition of the grid, the average vortex intensity at each section of the inlet of the straight pipe section of the cold fluid is greatly reduced from $4.244 / \mathrm{s}$ to $0.803 / \mathrm{s}$, which indicates the significant decrease of $81.1 \%$.

\section{Effect of Grid on Deformation of the Heat Exchange Tube Bundle}

When the diversion grid is not arranged, the heat exchange tube bundle near the inside of the baffle plate heat exchanger has the strongest backflow and the largest vortex intensity, which may affect the performance of the heat exchange tube bundle. Therefore, the deformation of the heat exchange tube is calculated.

Figure 3A shows the deformation of the heat exchange tube bundle in $Y, Z$, and $X$ directions without grid arrangement. The deformation in the $Y$ direction is the largest, about $85 \mathrm{~mm}$, which is due to the linear expansion and elongation caused by the temperature of the fluid. In the $Z$ direction (windward direction), the maximum deformation is $1.39 \mathrm{~mm}$, which occurs at the position of the strongest backflow. The deformation in the $X$-direction is reduced from 1.04 to $0.7 \mathrm{~mm}$ with the reduction of $32.7 \%$. The maximum deformation in the $X$-direction is also found at the strongest point of the vortex.

The deformation of the heat exchange tube bundle after the diversion grid is arranged can be seen in Figure 3B. As can be seen from the figure, the tube bundle has no significant changes in the $Y$ direction compared with that without the grid. The maximum deformation in the $Z$ direction is $1.03 \mathrm{~mm}$, which is $26 \%$ less than that without the grid. When the flow field is improved, the flow velocity of the fluid at the location of the strong vortex is decreased with the reduction of the partial velocity and the deformation in the $X$ direction. Therefore, the deformation in both $X$ and $Z$ directions is significantly reduced with the improvement of the flow field by arranging the grid. It can be seen that by arranging the grid to improve the flow field at the cold fluid inlet, the deformation of the heat exchange tube in the windward direction can be effectively reduced, the influence of reflux on the performance of the bundle can be reduced, and the safety of the device can be improved.

\section{CONCLUSION}

In this study, the distribution of the flow field and deformation of the tube bundle of the baffle plate heat exchanger with or without grid are calculated, respectively, and the influence of grid arrangement on the flow field and tube bundle performance is studied. The following conclusions can be drawn:

(1) After the cold fluid from the trumpet into the heat exchanger, it will form the phenomenon of the jet flow due to the sudden increase of the cross-section area, resulting in the backflow of the fluid.

(2) The fluid can be effectively diverted, and the reflux situation can be improved through the arrangement of the middle dense spacing on both sides of the sparse spacing grid. The average vortex intensity at the middle section of the inlet of the straight pipe section of the cold fluid decreased from $4.244 / \mathrm{s}$ to $0.803 / \mathrm{s}$ after the arrangement of the grid arrangement, which decreased by $81.1 \%$.

(3) By arranging the grid to improve the flow field at the cold fluid inlet trumpet, the deformation of the heat exchange tube in the windward direction can be reduced by $26 \%$, and the safety of the device can be improved.

\section{DATA AVAILABILITY STATEMENT}

The original contributions presented in the study are included in the article/Supplementary Material; further inquiries can be directed to the corresponding author.

\section{AUTHOR CONTRIBUTIONS}

ML ,ZF, YW, HY, WX, HZ, and DH contributed to conception and design of the study, ML performed the simulations and wrote the first draft of the manuscript. All authors contributed to manuscript revision,read, and approved the submitted version. 


\section{REFERENCES}

Blecich, P. (2015). Experimental Investigation of the Effects of Airflow Nonuniformity on Performance of a Fin-And-Tube Heat Exchanger. Int. J. Refrigeration. 59, 65-74. doi:10.1016/j.ijrefrig.2015.06.029

Chen, S. S. (1987). A General Theory for Dynamic Instability of Tube Arrays in Crossflow. J. Fluids Structures. 1 (1), 35-53. doi:10.1016/S0889-9746(87)90170-8

Liu, Y. H., Zhou, Y. P., Peng, C., Li, J. W., and Ren, H. L. (2017). Effect of Splitters and Air Distributor in Variable Cross-Section Flueon Reheater Inlet Flow Field. Sci. Technology Eng. 17, 356-362. doi:10.3969/j.issn.1671-1815.2017.32.058

Long, J. Y. (2014). Design of Boiler Economizer and Simulation of Flow Field about Uniformity. [master's Thesis]. Shanghai: Donghua University.

Miao, Q. (2016). The Study on Fluid Induced Vibration of Tube Bundles in Heat Exchanger. [master's Thesis]. Dalian: Dalian University of Technology.

Shi, Q. H., Su, W. H., and Wang, H. G. (2019). Characteristic of Fluid Induced Vibration of Tube Bundle with Realistic Flow Field. J. Vib Eng. Technol. 32 (02), 278-287. doi:10.16385/j.cnki.issn.1004-4523.2019.02.011

Wang, R. (2015). The Numerical Simulation of Heat Exchange Tube Bundle Based on thermal Fluid-Structural Interaction. [master's Thesis]. Dalian: Dalian University of Technology.

Wu, H., Wei, H. Q., Zhou, S., and Zhao, C. H. (2020). Design Optimization of the MGGH Flue System for 600MW Coal-Fired Units. Power equipment. 34 (04), 262-267+278. doi:10.19806/j.cnki.fdsb.2020.04.009

Xu, S. H. (1999). Method for Direct Calculation of Flue Gas Properties. J. Suzhou Silk Inst. Technology. 19 (3), 32-36.
Yang, J., Liu, J., Liu, X. H., Xie, T., and Liao, Z. Y. (2019). Optimization Design of Tubular Gas Gas Heater. Proc. CSEE. 39, 2384-2393. doi:10.13334/j.02588013.pcsee. 181640

Conflict of Interest: Authors ML, ZF, YW, HY, WX, and HZ were employed by the company Everbright Environmental Technology (China) Co.,LTD.

The remaining author declares that the research was conducted in the absence of any commercial or financial relationships that could be construed as a potential conflict of interest.

Publisher's Note: All claims expressed in this article are solely those of the authors and do not necessarily represent those of their affiliated organizations, or those of the publisher, the editors, and the reviewers. Any product that may be evaluated in this article, or claim that may be made by its manufacturer, is not guaranteed or endorsed by the publisher.

Copyright (c) 2022 Lu, Feng, Wang, Yang, Xia, Zhu and Hua. This is an openaccess article distributed under the terms of the Creative Commons Attribution License (CC BY). The use, distribution or reproduction in other forums is permitted, provided the original author(s) and the copyright owner(s) are credited and that the original publication in this journal is cited, in accordance with accepted academic practice. No use, distribution or reproduction is permitted which does not comply with these terms. 